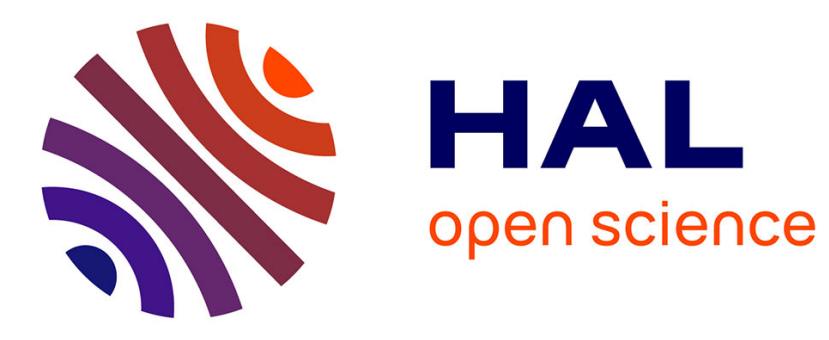

\title{
Qualitative Integrals and Desintegrals: How to Handle Positive and Negative Scales in Evaluation
}

Didier Dubois, Henri Prade, Agnés Rico

\section{To cite this version:}

Didier Dubois, Henri Prade, Agnés Rico. Qualitative Integrals and Desintegrals: How to Handle Positive and Negative Scales in Evaluation. 14th International Conference on Information Processing and Management of Uncertainty in Knowledge-based Systems (IPMU 2012), Jul 2012, Catane, Italy. pp.306-316, 10.1007/978-3-642-31718-7_32. hal-03344986

\section{HAL Id: hal-03344986 \\ https://hal.science/hal-03344986}

Submitted on 15 Sep 2021

HAL is a multi-disciplinary open access archive for the deposit and dissemination of scientific research documents, whether they are published or not. The documents may come from teaching and research institutions in France or abroad, or from public or private research centers.
L'archive ouverte pluridisciplinaire HAL, est destinée au dépôt et à la diffusion de documents scientifiques de niveau recherche, publiés ou non, émanant des établissements d'enseignement et de recherche français ou étrangers, des laboratoires publics ou privés. 


\title{
Qualitative Integrals and Desintegrals: How to Handle Positive and Negative Scales in Evaluation
}

\author{
Didier Dubois ${ }^{1}$, Henri Prade ${ }^{1}$, and Agnès Rico ${ }^{2}$ \\ 1 IRIT, CNRS and Université de Toulouse, France \\ \{dubois, prade\}@irit.fr \\ 2 ERIC, Université de Lyon, France \\ agnes.rico@univ-lyon1.fr
}

\begin{abstract}
Integrals are currently used in multiple criteria analysis for synthesizing into a global evaluation the advantages possessed by a potential choice. As such, integrals are operators that increase with the criteria evaluations. However, an item may be also evaluated in terms of its defects. Then the more and the greater the defects, the smaller the evaluation should be. An operator that can provide a synthesis of the defects of an item in this sense is called a desintegral. Desintegrals are maximal when no defects at all are present, while integrals are maximal when all advantages are sufficiently present. So, the greater the value of an integral, or a desintegral, the better the corresponding item since advantages are greater, or defects are smaller respectively. Desintegrals implicitly refer to a negative scale, since an order-reversing mapping of the scale used for evaluating each criterion transforms the degree to which the value is advantageous into a degree to which it is disadvantageous, and conversely. In this paper, we provide an organised description of counterparts to Sugeno integrals that synthesize positive or negative evaluations in the qualitative framework of a totally ordered residuated lattice equipped with an involutive negation. We exploit three kinds of criteria weighting schemes that are allowed by this algebraic structure.
\end{abstract}

\section{Introduction}

Choquet integrals and Sugeno integrals are widely used in multiple criteria aggregation, respectively in quantitative and in qualitative settings [9]. Roughly speaking, quantitative integration amounts to cumulate elementary evaluations in order to build a global one, and any strict increase of these evaluations lead to a cumulative effect as expected. In the qualitative setting, Sugeno integrals rather provide a synthesis of the elementary evaluations, and are only increasing in a broad sense. Thus, using integrals, we start from the bottom / minimal value of any evaluation, say 0 , and cumulate or synthesize elementary evaluations, which have thus a positive flavor, as contributions to the global evaluation.

Desintegration is the converse. We start with the top/ maximal evaluation, say 1 , and each time a negative feature is reported, it contributes to diminish 
the global evaluation. In the negative evaluation framework, we call the counterpart of a Sugeno integral a desintegral. With the idea of desintegrals, we try to model evaluations such that if an object has some properties, then its evaluation becomes worse. More generally, the better a feature is satisfied, the worse the global evaluation. So the features, properties or criteria have a negative flavor.

Moreover, the form of the aggregation operation is affected by the way the criteria weights modify the local evaluations, and the pessimistic or optimistic nature of the evaluation. So there may exist variants of qualitative aggregation techniques that differ from Sugeno integrals not only due the polarity of the involved scales, but also in the handling of the importance weights.

The paper is structured as follows. The next section is devoted to the description of the considered landscape of aggregation operations, and their motivations. Section 3 focuses on the properties of one example of residuated implicationbased counterpart of Sugeno integrals, that combines qualitative ratings in negative scales and construct a gobal rating on a positive scale. In the conclusion we mention some open problems and briefly discuss the joint use of Sugeno integrals and the Sugeno desintegrals when some criteria have a positive flavor and others have a negative flavor.

\section{General Setting and Motivation}

In a bipolar evaluation framework one may handle reasons in favor of an alternative and reasons against it. In most situations, criteria are evaluated on positive scales and the resulting aggregation is also valued on a positive scale. Namely assuming this scale is the unit interval, the bottom 0 is viewed as worse than the top 1 and the aggregation is monotonically increasing. However one may consider aggregating negative criteria, that is, features that are to be explicitly avoided. In that case the local evaluation scales are negative ( 0 is better than 1 ), and if the global scale is a positive one, the aggregation operation needs to be monotonically decreasing. The more fulfilled are the negative features, the smaller is the global evaluation.

\subsection{Algebraic Framework}

Let us consider a set of criteria $\mathcal{C}=\left\{C_{1}, \ldots, C_{n}\right\}$. Some objects or acts are evaluated according to the criteria. The evaluation scale associated to each criterion is a totally ordered scale $L$, for instance a finite one or $[0,1]$. In such a context an object is viewed as a function $f$ from $\mathcal{C}$ to $L$, encoded as a vector $\left(f_{1}, \ldots, f_{n}\right) \in[0,1]^{n} . f_{i}$ is the evaluation of $f$ according to the criterion $C_{i}$.

We consider $L$ as a Heyting algebra, i.e., a complete residuated lattice with top 1 and bottom 0 . More precisely, $<L, \wedge, \vee, \rightarrow, 0,1>$ is a complete lattice. Of course, $\langle L, \wedge, 1\rangle$ is a commutative monoid (i.e $\wedge$ is associative, commutative and for all $a \in L a \wedge 1=a)$. The operator $\rightarrow$ called the residuum is such that $(a \rightarrow b) \wedge a \leq b$, and moreover $a \rightarrow b=\sup \{c: a \wedge c \leq b\}$. In such a case $\rightarrow$ is Gödel implication. Let us present some basic properties of residuated lattices useful in the sequel: For all $a, b, c \in L$, 
$-a \leq b$ if and only if $a \rightarrow b=1$;

$-a \rightarrow a=1, a \rightarrow 1=1,0 \rightarrow a=1,1 \rightarrow a=a$;

$-a \leq b$ entails $c \rightarrow a \leq c \rightarrow b$ and $b \rightarrow c \leq a \rightarrow c$.

The adjointness property reads: $a \leq b \rightarrow c$ if and only if $a \wedge b \leq c$. Moreover as $L$ is a totally ordered set the prelinearity property: for all $a, b,(a \rightarrow b) \vee(b \rightarrow a)=1$ is always satisfied.

In order to handle the polarity of the evaluation scale, we also need an orderreversing operation on $L$, denoted by $1-\cdot$, that is decreasing and involutive (a Kleene negation). If $L$ is a positive scale ( 1 means good, 0 means bad), then $\{1-a: a \in L\}$ is a negative scale (1 means bad, 0 means good). On a complete residuated lattice, a negation is defined by $\neg a=a \rightarrow 0$ such that $\neg a=1$ if $a=0$ and 0 otherwise, hence not involutive. This intuitionistic negation clearly differs from the Kleene negation.

Overall the structure $\langle L, \wedge, \vee, \rightarrow, 1-\cdot, 0,1\rangle$ is a complete De Morgan residuated lattice, since $\langle L, \wedge, \vee, 1-\cdot, 0,1>$ is a De Morgan algebra. We consider such a framework because of its qualitative nature that fits the requirements of Sugeno integral.

\subsection{Aggregation and Scale Polarity}

Due to the polarity of the evaluation scales, there are 4 cases to be considered for an aggregation operation $L_{1} \times \ldots \times L_{n} \mapsto L$ :

1. Scales $L_{i}$ and $L$ are positive (satisfaction).

2. Scales $L_{i}$ are negative (dissatisfaction) and $L$ is positive (satisfaction).

3. Scales $L_{i}$ are positive (satisfaction) and $L$ is negative (dissatisfaction).

4. Scales $L_{i}$ and $L$ are negative (dissatisfaction).

In the first case of positive scales, there are two elementary qualitative aggregation schemes that make sense: $\wedge_{i=1}^{n} f_{i}$ if one is very demanding, and $\vee_{i=1}^{n} f_{i}$ if one fulfilled criterion is enough.

In the second case where negative ratings are merged on a positive scale, the value $x_{i}$ is all the greater as the evaluation is bad, and the global score is all the greater as the resulting evaluation is better. The counterpart of these two elementary aggregations can be handled by first reversing the negative scales and then aggregating the results as previously, or on the contrary aggregating the negative scores and reversing the global result:

- The demanding case reads: $\wedge_{i=1}^{n}\left(1-f_{i}\right)=1-\vee_{i=1}^{n} f_{i}$

- The loose global evaluation reads $\vee_{i=1}^{n}\left(1-f_{i}\right)=1-\wedge_{i=1}^{n} f_{i}$

Note that when aggregating negative scores $\vee$ is demanding while $\wedge$ is loose. Then, the aggregation operation is monotonically decreasing. The two other cases can be discussed likewise. We now successively examine qualitative weighted aggregations in cases 1 and 2, with positive and then negative input scales. In both cases, the global evaluation lies in a positive scale. 


\subsection{Three Qualitative Weighted Aggregations for Positive Scales}

Let $\pi_{i}$ be the importance level of criterion $i$, valued on a positive scale. Namely, $\pi_{i}$ is all the greater as criterion $i$ is important. These levels are not used in the same way in a loose or a demanding aggregation. Moreover they may alter the local evaluations $f_{i}$ in at least three manners.

Saturation Levels. The most well-known cases for positive criteria are when the importance weights act as saturation levels. The corresponding prioritized aggregation schemes are of the form $S L M A X_{\pi}(f)=\vee_{i=1}^{n} \pi_{i} \wedge f_{i}$ (prioritized maximum) and $S L M I N_{\pi}(f)=\wedge_{i=1}^{n}\left(1-\pi_{i}\right) \vee f_{i}$ (prioritized minimum). They are special cases of Sugeno integral, where the weights $\mu(A)$ generalize importance weights $\pi_{i}$ to groups of criteria $A \subseteq \mathcal{C}$. The fuzzy measure $\mu: 2^{\mathcal{C}} \rightarrow[0,1]$ is an increasing set function such that $\mu(\emptyset)=0$ and $\mu(\mathcal{C})=1$, as larger groups of criteria are more important than smaller ones. The Sugeno integral of $f$ with respect to $\mu$ is denoted by $\oint_{\mu}(f)=\vee_{A \subseteq \mathcal{C}} \mu(A) \wedge \wedge_{i \in A} f_{i}{ }^{1}$ If all local evaluations $f_{i}$ equal a constant $a$ then $\oint_{\mu}(f)=a$. The prioritized maximum and prioritised minimum are recovered if $\mu$ is a possibility or a necessity measure respectively.

Softening Thresholds. Another approach to the weights $\pi_{i}$ is to consider them as softeners that make local evaluations less demanding. One may for instance, consider that $f_{i} \geq \pi_{i}$ is enough to reach full satisfaction. Otherwise one sticks to evaluation $f_{i}$. More precisely, such a modified satisfaction is computed with the Gödel implication: $\pi_{i} \rightarrow f_{i}$ where $x \rightarrow y=\left\{\begin{array}{c}1 \text { if } x \leq y \\ y \text { otherwise }\end{array}\right.$. Note that $\pi_{i}=0$ represents such a high tolerance as to consider the criterion to be ever fulfilled. This thresholding scheme is easily applied to the demanding aggregation in the form $S T M I N_{\pi}(f)=\wedge_{i=1}^{n} \pi_{i} \rightarrow f_{i}$. Note that in the previous prioritization scheme $S L M A X_{\pi}(f)=1-S L M I N_{\pi}(1-f)$. Preserving this connection leads to define the loose counterpart of the $S T M I N_{\pi}$ connective as $S T M A X_{\pi}(f)=\vee_{i=1}^{n} \pi_{i} \star f_{i}$ where $x \star y=1-x \rightarrow(1-y)=\left\{\begin{array}{c}0 \text { if } x \leq 1-y \\ y \text { otherwise }\end{array}\right.$. This non commutative conjunction was introduced in [4]. In the more general case of weighting groups of criteria by means of a monotonic increasing setfunction $\mu$, a residuated implication-based counterpart of a Sugeno integral is of the form

$$
\oint_{\mu}^{\uparrow}(f)=\wedge_{A \subseteq \mathcal{C}} \mu(A) \rightarrow \vee_{i \in A} f_{i}
$$

Proposition 1. If $\mu$ is a possibility measure, then $\oint_{\mu}^{\uparrow}(f)=\operatorname{STMIN}_{\pi}(f)$.

\footnotetext{
1 This notation whereby the capacity appears as a subscript is unusual for integrals. It is conveniently concise for this paper where the domain plays no particular role.
} 
Proof. Suppose $\mu=\Pi$ based on the possibility degrees $\pi_{i}$. It is obvious that $\oint_{\Pi}^{\uparrow}(f) \leq S T M I N_{\pi}(f)$ as the former considers the infimum over many more situations. Now let $A$ be a set such that $\oint_{\Pi}^{\uparrow}(f)=\max _{j \in A} \pi_{j} \rightarrow \vee_{i \in A} f_{i}$. Let $k, \ell$ such that $\oint_{\Pi}^{\uparrow}(f)=\pi_{k} \rightarrow f_{\ell}$. If $\pi_{k} \rightarrow f_{\ell}=1$, then $\oint_{\Pi}^{\uparrow}(f) \geq \operatorname{STMIN}(f)$ is obvious. Otherwise $\pi_{k} \rightarrow f_{\ell}<1$. But by construction $f_{\ell} \geq f_{k}$. Hence $\oint_{\Pi}^{\uparrow}(f)=f_{\ell} \geq \pi_{k} \rightarrow f_{k} \geq S T M I N_{\pi}(f) . \mathrm{QED}$.

The extension of the $S T M A X_{\pi}$ aggregation is $\oint_{\mu}^{r \star}(f)=\vee_{A \subseteq \mathcal{C}} \mu(A) \star \wedge_{i \in A} f_{i}$.

Drastic Thresholdings. Note that $\operatorname{STMIN}_{\pi}(f)$ cannot be considered as a proper generalization to fuzzy events of a necessity measure, since when the $f_{i}$ 's belong to $\{0,1\}$, we do not get $\operatorname{STMIN}_{\pi}(f)=N(A)=\min _{i \notin A}\left(1-\pi_{i}\right)$ for $A=\left\{i \mid f_{i}=1\right\}$. It is known [5] that the natural extension of necessity measures to fuzzy events based on Gödel implication is $D T M I N_{\pi}(f)=\min _{i}\left(1-f_{i}\right) \rightarrow$ $\left(1-\pi_{i}\right)$. The effect of the weight $\pi_{i}$ on the rating $f_{i}$ is as follows: if $f_{i} \geq \pi_{i}$, the rating becomes maximal, i.e. 1 , otherwise it is always turned into $1-\pi_{i}$. Two remarks are worth stating. First, the local rating scale reduces to the binary scale $\left\{1-\pi_{i}, 1\right\}$. Second, if $\pi_{i}$ is high, the local rating is drastically downgraded to $1-\pi_{i}$. The loose counterpart of the $D T M I N_{\pi}$ connective is $D T M A X_{\pi}(f)=$ $\vee_{i=1}^{n} f_{i} \star \pi_{i}$ where $\star$ is the same non-commutative conjunction as above. The extension of the $D T M I N_{\pi}$ aggregation to weighting groups of criteria is of the form (where $\bar{A}$ is the complement of $A$ )

$$
\oint_{\mu}^{\Uparrow}(f)=\wedge_{A \subseteq \mathcal{C}}\left(\wedge_{i \in A}\left(1-f_{i}\right) \rightarrow \mu(\bar{A})\right) .
$$

Proposition 2. if $\mu$ is a necessity measure based on $\pi$, then $\oint_{\mu}^{\Uparrow}(f)=D T M I N_{\pi}(f)$.

The extension of the DTMAX $X_{\pi}$ aggregation is $\oint_{\mu}^{l \star}(f)=\vee_{A \subseteq \mathcal{C}} \wedge_{i \in A} f_{i} \star \mu(A)$.

\subsection{Three Qualitative Weighting Methods for Negative Scales}

Let us turn to the case where we rate local features on negative scales and get a global evaluation on a positive scale. Under this convention, a value $t_{i}$ acts as a tolerance or permissiveness level on a negative scale: the higher $t_{i}$, the less important criterion $i$. Then the threshold scheme for negative criteria goes as follows: the tolerance level $t_{i}$ now serves to turn negative flexible ratings ( $f_{i}$ now represents a degree of defect or violation, where the greater $f_{i}$, the worse the rating) into positive ones that are further aggregated on a positive scale. There are again three qualitative aggregation schemes that reflect the three cases encountered in the positive case. They are called desintegrals since they will involve decreasing set functions $\nu$, called a fuzzy antimeasure: It is a decreasing set function $\nu: 2^{\mathcal{C}} \rightarrow[0,1]$ such that $\nu(\emptyset)=1$ and $\nu(\mathcal{C})=0$. 
Saturation Levels. The result of applying tolerance $t_{i}$ to the negative rating $f_{i}$ results in a positive rating that cannot be below $t_{i}$. Moreover the local rating scale is reversed, which leads to a local positive rating $\left(1-f_{i}\right) \vee t_{i}$. The corresponding demanding aggregation scheme is $S L M I N_{t}^{n e g}(f)=\wedge_{i=1}^{n}\left(1-f_{i}\right) \vee t_{i}$, while the loose one is of the form $S L M A X_{t}^{n e g}(f)=\vee_{i=1}^{n}\left(1-t_{i}\right) \wedge\left(1-f_{i}\right)$. Note that $S L M I N_{t}^{n e g}(f)=S L M I N_{1-\pi}(1-f)$ and $S L M A X_{t}^{n e g}(f)=S L M A X_{1-\pi}(1-f)$. The corresponding extension of $S L M A X_{t}^{\text {neg }}$ based on a monotonically decreasing function $\nu$ to the weighting of groups of defects is given by the expression

$$
\oint_{\nu}(f)=\vee_{A \subseteq \mathcal{C}}\left(\nu(\bar{A}) \wedge \wedge_{i \in A}\left(1-f_{i}\right)\right)
$$

where we recognize the formal Sugeno integral $\oint_{\nu(\cdot)}(1-f)$. Morover $\oint_{\nu}(f)=$ $S L M A X_{t}^{n e g}(f)$ if $\nu(A)=\max _{i \in \bar{A}}\left(1-t_{i}\right)$.

Softening Thresholds. Here $t_{i}$ is viewed as a tolerance threshold such that it is enough to have $f_{i} \leq t_{i}$ (i.e. the defect rating remains smaller than the threshold) for the requirement to be totally fulfilled. Recall that now the requirement is to avoid defects. If the object possesses the defect to an extent higher than $t_{i}$, then the rating value is reversed, leading to a poor positive local rating. This weighting scheme is captured by the formula $\left(1-t_{i}\right) \rightarrow\left(1-f_{i}\right)$ where $\rightarrow$ is Gödel implication. This thresholding scheme is easily applied to the demanding aggregation in the form $S T M I N_{t}^{n e g}(f)=\wedge_{i=1}^{n}\left(1-t_{i}\right) \rightarrow\left(1-f_{i}\right)$. We can define the loose counterpart of the $S T M I N_{t}^{n e g}$ connective as $S T M A X_{t}^{n e g}(f)=\vee_{i=1}^{n}\left(1-t_{i}\right) \star\left(1-f_{i}\right)$ using the non commutative conjunction introduced before. In the more general case of weighting groups of defects by means of a monotonic decreasing set-function $\nu$, a residuated implication-based desintegral that generalizes $S T M I N_{t}^{n e g}$ is of the form

$$
\oint_{\nu}^{\downarrow}(f)=\wedge_{A \subseteq \mathcal{C}}\left((1-\nu(A)) \rightarrow \vee_{i \in A}\left(1-f_{i}\right)\right) .
$$

It is easy to check that $\oint_{\nu}^{\downarrow}(f)=\oint_{1-\nu}^{\uparrow}(1-f)$.

Proposition 3. If $\nu(A)=\Delta(A)=\wedge_{i \in A} t_{i}$, then $\oint_{\nu}^{\downarrow}(f)=S T M I N_{t}^{n e g}(f)$.

The antimeasure $\Delta$ is known as a guaranteed possibility measure [5]. Moreover, the corresponding extension of $S T M A X_{t}^{\text {neg }}$ is the desintegral

$$
\oint_{1-\nu}^{r \star}(1-f)=\vee_{A \subseteq \mathcal{C}}\left((1-\nu(A)) \star \wedge_{i \in A}\left(1-f_{i}\right)\right) .
$$

Drastic Thresholdings. The last weighting scheme can be described as follows. If $f_{i}>t_{i}$ then the local rating is considered bad and the (positive) result is downgraded to $t_{i}$. If $f_{i} \leq t_{i}$ then the local rating is fine and the (positive) result is 1 . It corresponds again to using Gödel implication and now computing $f_{i} \rightarrow t_{i}$. Note that $t_{i}=0$ means complete intolerance with respect to the slightest possession of defect $i$. Demanding that no bad feature be satisfied leads to a demanding 
aggregation of positive ratings $f_{i} \leq t_{i}$. This is $\operatorname{DTMIN}_{t}^{n e g}(f)=\wedge_{i=1}^{n} f_{i} \rightarrow t_{i}$, that is a guaranteed possibility function $\Delta$ applied to fuzzy event $f[5]$. Note that the local negative rating scale is changed into a binary positive scale $\left\{t_{i}, 1\right\}$. The loose counterpart of $D T M I N_{t}^{n e g}$ is $D T M A X_{t}^{n e g}=\vee_{i=1}^{n}\left(1-f_{i}\right) \star\left(1-t_{i}\right)$. The extension of the $D T M I N_{t}^{n e g}$ aggregation to tolerance levels attached to groups of defects corresponds to the following desintegral with respect to antimeasure $\nu$ is given by

$$
\oint_{\nu}^{\Downarrow}(f)=\wedge_{A \subseteq \mathcal{C}} \wedge_{i \in A} f_{i} \rightarrow \nu(A),
$$

with the following convention: $\wedge_{i \in \emptyset} f_{i}=0$ (otherwise, one must restrict the above expression to non-empty sets).

Proposition 4. If $\nu(A)=\Delta(A)=\wedge_{i \in A} t_{i}$, then $\oint_{\nu}^{\Downarrow}(f)=D T M I N_{t}^{n e g}(f)$.

Proof. According to the definitions we have $\oint_{\nu}^{\Downarrow}(f) \leq D T M I N_{t}^{n e g}(f)$, so let us prove that $D T M I N_{t}^{n e g}(f) \leq \oint_{\nu}^{\Downarrow}(f)$. Let $A^{*}$ be the set associated to the minimum in the definition of $\oint_{\nu}^{\Downarrow}(f)$, i.e., $\oint_{\nu}^{\Downarrow}(f)=\wedge_{i \in A^{*}} f_{i} \rightarrow \wedge_{i \in A^{*}} t_{i}$. The only possible values are 1 and $\wedge_{i \in A^{*}} t_{i}$. On both sides, we can have different criteria associated to the minimum. We denote them by $j$ and $k$, i.e., $\oint_{\nu}^{\Downarrow}(f)=f_{j} \rightarrow t_{k}$. If $\oint_{\nu}^{\Downarrow}(f)=1$, there is no difficulty. If $\oint_{\nu}^{\Downarrow}(f)<1$, then $f_{j}>t_{k}$. As $f_{j}=\wedge_{x \in A^{*}} f_{i}$ we have $f_{k} \geq f_{j}>t_{k}$. So $f_{j} \rightarrow t_{k}=f_{k} \rightarrow t_{k} \geq \inf _{i \in \mathcal{C}}\left(f_{i} \rightarrow t_{i}\right)$. So $\oint_{\nu}^{\Downarrow}(f) \geq$ $\operatorname{DTMIN}_{t}^{n e g}(f)$.

The extension of $D T M A X_{t}^{n e g}$ is $\oint_{\nu}^{l \star}(1-f)=\vee_{A \subseteq \mathcal{C}} \wedge_{i \in A}\left(1-f_{i}\right) \star \nu(A)$.

The above survey can be summarized using the following terminology: we call (qualitative) integral (resp. desintegral) a weighted aggregation operation that is increasing (resp. decreasing) with respect to the local ratings. We call an integral or a desintegral conjunction-based (resp. implication-based) when it takes the form of a maximum (resp. minimum) of the conjunction of (resp. implication between) weights and ratings. We call an integral or a desintegral drastic (resp. soft) when the result can only belong to the set of weights, making the local rating scale binary (resp. when the result belongs to the original rating scale or its reverse). In the following we more particularly study drastic desintegrals with respect to general set-functions. Indeed, one may find situations where the weighting function is nonmonotonic. For instance, if good objects are those that possess exactly $k$ properties out of $n>k$.

\section{Properties of the Drastic Desintegrals}

In this section, we consider, as an example, the properties of drastic desintegrals $\oint_{\sigma}^{\Downarrow}(f)$, where $\sigma: 2^{\mathcal{C}} \rightarrow[0,1]$ is a general set-function. The name desintegral is justified by the following

Proposition 5. If $f \leq g$ then $\oint_{\sigma}^{\Downarrow}(g) \leq \oint_{\sigma}^{\Downarrow}(f)$. 
The Sugeno integral $\oint_{\mu}$ with respect to a fuzzy measure $\mu$ is an aggregation function satisfying the following properties.

Proposition 6. For all functions $f, g$ and for all $a$ in $[0,1]$,

$$
\begin{aligned}
& \oint_{\mu}(f \wedge g) \leq \oint_{\mu}(f) \wedge \oint_{\mu}(g), \quad \oint_{\mu}(f \vee g) \geq \oint_{\mu}(f) \vee \oint_{\mu}(g) \\
& \oint_{\mu}(a \wedge f)=a \wedge \oint_{\mu}(f), \quad \oint_{\mu}(a \rightarrow f) \leq a \rightarrow \oint_{\mu}(f) .
\end{aligned}
$$

As may be expected the drastic desintegrals satisfy similar but opposite properties. More precisely the following result, proved in [6] for an MV-algebra with an antimeasure, is still satisfied in our context.

Proposition 7. For all functions $f, g$ and for all $a$ in $[0,1]$,

$$
\begin{aligned}
& \oint_{\sigma}^{\Downarrow}(f \wedge g) \geq \oint_{\sigma}^{\Downarrow}(f) \vee \oint_{\sigma}^{\Downarrow}(g), \quad \oint_{\sigma}^{\Downarrow}(f \vee g) \leq \oint_{\sigma}^{\Downarrow}(f) \wedge \oint_{\sigma}^{\Downarrow}(g) \\
& \oint_{\sigma}^{\Downarrow}(a \wedge f) \leq a \rightarrow \oint_{\sigma}^{\Downarrow}(f), \quad \oint_{\sigma}^{\Downarrow}(a \rightarrow f) \geq a \wedge \oint_{\sigma}^{\Downarrow}(f) .
\end{aligned}
$$

In order to use the drastic desintegrals we need to understand how the set functions used can be interpreted.

Proposition 8. For all $A \subseteq \mathcal{A}, \sigma(A)$ represents an upper bound of the global satisfaction rating that an object can receive with a drastic desintegral if it satisfies all properties in the group $A$ to a degree higher than $\sigma(A)$.

Proof. Since the hypothesis implies $\wedge_{C_{i} \in A} f_{i}>\sigma(A), \wedge_{C_{i} \in A} f_{i} \rightarrow \sigma(A)=\sigma(A)$, which entails $\oint_{\sigma}^{\Downarrow}(f) \leq \wedge_{C_{i} \in A} f_{i} \rightarrow \sigma(A)$.

Example 1. We consider three criteria $C_{1}, C_{2}, C_{3}$. We want to select objects that satisfy only $C_{1}$, only $C_{2}$ or only $C_{3}$. We consider $\sigma$ defined as follows $\sigma\left(C_{1}\right)=$ $\sigma\left(C_{2}\right)=\sigma\left(C_{3}\right)=1 ; \sigma$ is 0 otherwise.

Let us compute $\oint_{\sigma}^{\Downarrow}(1,0,0)$ : If $A \neq\left\{C_{1}\right\}$ then $\wedge_{C_{i} \in A} f_{i}=0$ and $\wedge_{C_{i} \in A} f_{i} \rightarrow$ $\sigma(A)=1$. If $A=\left\{C_{1}\right\}$ then $\wedge_{C_{i} \in A} f_{i} \rightarrow \sigma(A)=1 \rightarrow 1=1$. So we have $\oint_{\sigma}^{\Downarrow}(1,0,0)=1$. Similarly we have $\oint_{\sigma}^{\Downarrow}(0,1,0)=\oint_{\sigma}^{\Downarrow}(0,0,1)=1$.

Let us compute $\oint_{\sigma}^{\Downarrow}(1,1,0)$ : If $A=\left\{C_{1}, C_{2}\right\}$ then $\wedge_{C_{i} \in A} f_{i}=1$ and $\wedge_{C_{i} \in A} f_{i} \rightarrow$ $\sigma(A)=1 \rightarrow 0=0$. So we have $\oint_{\sigma}^{\Downarrow}(1,1,0)=0$. Similarly we have $\oint_{\sigma}^{\Downarrow}(1,0,1)=$ $\oint_{\sigma}^{\Downarrow}(0,1,1)=\oint_{\sigma}^{\Downarrow}(0,1,1)=0$.

In the above example, if we consider $\sigma$ such that $\sigma\left(C_{1}\right)=\sigma\left(C_{2}\right)=\sigma\left(C_{3}\right)=0.5$ and $\sigma$ is 0 otherwise then $\oint_{\sigma}^{\Downarrow}(1,0,0)=\oint_{\sigma}^{\Downarrow}(0,1,0)=\oint_{\sigma}^{\Downarrow}(0,0,1)=0.5$. Also we have $\oint_{\sigma}^{\Downarrow}(1,1,0)=\oint_{\sigma}^{\Downarrow}(1,0,1)=\oint_{\sigma}^{\Downarrow}(0,1,1)=0$.

The global evaluation of an object calculated with a drastic desintegral, belongs to the interval $[0,1]$. In order to interpret the obtained result, for example in order to select objects, we need to identify the objects that obtain the best evaluation and those that receive the worst one.

Proposition 9. $\oint_{\sigma}^{\Downarrow}(f)=1$ if and only if $\forall A, \exists C_{i} \in A$ such that $f_{i} \leq \sigma(A)$. $\oint_{\sigma}^{\Downarrow}(f)=0$ if and only if $\exists A$ such that $\sigma(A)=0$ and $\forall C_{i} \in A, f_{i}>0$. 
Proof. $\oint_{\sigma}^{\Downarrow}(f)=1$ if and only if $\forall A, \wedge_{C_{i} \in A} f_{i} \rightarrow \sigma(A)=1$ i.e. $\forall A, \wedge_{C_{i} \in A} f_{i} \leq$ $\sigma(A)$, which is equivalent to $\exists C_{i} \in A$ such that $f_{i} \leq \sigma(A) . \oint_{\sigma}^{\Downarrow}(f)=0$ if and only if $\exists A$ such that $\wedge_{C_{i} \in A} f_{i} \rightarrow \sigma(A)=0$, i.e, $\sigma(A)=0$ and $\wedge_{C_{i} \in A} f_{i}>0$.

We conclude this section with mathematical properties classically studied for aggregation functions.

Proposition 10. $\oint_{\sigma}^{\Downarrow}(0, \ldots, 0)=1, \quad \oint_{\sigma}^{\Downarrow}(1, \ldots, 1)=\wedge_{A \subseteq \mathcal{C}} \sigma(A)$,

$\oint_{\sigma}^{\Downarrow}(c, \ldots, c)=\wedge_{\{A \mid \sigma(A)<c\}} \sigma(A)$, (with convention $\wedge_{A \in \emptyset} \sigma(A)=1$ ).

$\oint_{\sigma}^{\Downarrow}\left(1_{A}\right)=\wedge_{B \subseteq A} \sigma(B)$ (we denote the characteristic function of $A$ by $1_{A}$ ).

Proof. $\oint_{\sigma}^{\Downarrow}(0, \ldots, 0)=\wedge_{A \subseteq \mathcal{C}} 0 \rightarrow \sigma(A)=1, \oint_{\sigma}^{\Downarrow}(1, \ldots, 1)=\wedge_{A \subseteq \mathcal{C}} 1 \rightarrow \sigma(A)=$ $\wedge_{A \subseteq \mathcal{C}} \sigma(A) . \oint_{\sigma}^{\Downarrow}(c, \ldots, c)=\wedge_{A \subseteq \mathcal{C}} c \rightarrow \sigma(A)$ where $c \rightarrow \sigma(A)$ is 1 if $c \leq \sigma(A)$ and is $\sigma(A)$ otherwise.

If $B \nsubseteq A$ then $\wedge_{C_{i} \in B} 1_{A} \rightarrow \sigma(A)=0 \rightarrow \sigma(A)=1$.

If $B \subseteq A$, then $\wedge_{C_{i} \in B} 1_{A} \rightarrow \sigma(B)=1 \rightarrow \sigma(B)=\sigma(B)$.

If there exists $A$ such that $\sigma(A)=0$ then $\oint_{\sigma}^{\Downarrow}(1, \ldots, 1)=\oint_{\sigma}^{\Downarrow}(c, \ldots, c)=0$. More generally, if $\sigma$ is a fuzzy antimeasure then $\oint_{\sigma}^{\Downarrow}(0, \ldots, 0)=1, \oint_{\sigma}^{\Downarrow}(1, \ldots, 1)=$ $\oint_{\sigma}^{\Downarrow}(c, \ldots, c)=0$ and $\oint_{\sigma}^{\Downarrow}\left(1_{A}\right)=\sigma(A)$. Note that this particular case can be also proved using the results presented in [6].

\section{Concluding Remarks}

This paper outlines a framework for a class of general aggregation functions on a complete residuated, totally ordered set with an order-reversing negation. As some of them use residuated implications, they cannot be generated from standard Sugeno integrals and negation. A number of questions remain pending. For instance, Sugeno integral can be written in two equivalent ways as

$$
\oint_{\mu}(x)=\vee_{A \subseteq \mathcal{C}} \mu(A) \wedge \wedge_{i \in A} f_{i}=\wedge_{A \subseteq \mathcal{C}} \mu(\bar{A}) \vee \vee_{i \in A} f_{i}
$$

In the right-hand side expression we can recognise a Kleene-Dienes implicationbased qualitative integral $(a \longmapsto b=(1-a) \vee b)$ with respect to the conjugate set-function $\bar{\mu}(A)=1-\mu(\bar{A})$. Hence the natural question is whether this type of identity remains valid on our qualitative structure, for Gödel implication, and the corresponding non-commutative conjunction. Besides, other algebraic frameworks are worth studying from this perspective, like MV-algebras [6].

Another question is the simultaneous handling of positive and negative criteria for decision evaluation. Suppose the set of the criteria is divided into two parts: the set of positive criteria denoted $\mathcal{C}^{+}$and the set of negative criteria denoted $\mathcal{C}^{-}$ where $\mathcal{C}^{+} \cup \mathcal{C}^{-}=\mathcal{C}$ and $\mathcal{C}^{+} \cap \mathcal{C}^{-}=\emptyset$. On $\mathcal{C}^{+}$, a fuzzy measure $\mu$ represents the satisfactory level needed to have a good evaluation. Hence the global evaluation with respect to the positive criteria can be calculated using for example the 
Sugeno integral with respect to $\mu$ : $\oint_{\mu}$. On $\mathcal{C}^{-}$an antimeasure $\nu$, represents an upper bound of the global satisfaction level an object can receive if it satisfies all the properties with a level bigger than $\nu$. Hence the global evaluation with respect to the negative criteria can be computed using for example the drastic desintegral with respect to $\nu: \oint_{\nu}^{\Downarrow}$.

One may try to merge the positive evaluations obtained from a integral over positive criteria and a desintegral with respect to negative ones, or on the contrary handle them separately for making a final comparison of objects. We tend to favor a separate handling of positive and negative aspects, in contrast with other approaches like Cumulative Prospect Theory that are numerical, or the ones of Grabisch $[8,7]$ that try to work with a single qualitative bipolar scale where available combination of positive and negative values look debatable. See also [10]. On the contrary, we are more in the spirit of bivariate bipolar approaches to evaluation such as the ones proposed by Bonnefon and colleagues $[2,3]$. However, their approach is restricted to Boolean valuation scales (all-ornothing positive or negative criteria) and importance levels bear on single criteria. The framework presented in this paper opens the way to a generalization of qualitative bipolar decision evaluation to criteria with more refined value scales and generalized weightings of groups of criteria. Our bipolar approach is also somewhat similar to Atanassov "Intuitionistic Fuzzy Set" (AIFS) aggregation function theory[1], since it also handles pairs of positive and negative values. However, in the AIFS approach, a pair $(\mu, \nu)$ of membership and nonmembership values is such that $\mu+\nu \leq 1$ and is interpreted as an uncertainty gap of the form $[\mu, 1-\nu]$, while in our view the positive and negative evaluations are to be considered as two independent precise evaluations to be used conjointly.

\section{References}

1. Beliakov, G., Bustince, H., Goswami, D.P., Mukherjee, U.K., Pal, N.R.: On averaging operators for Atanassov's intuitionistic fuzzy sets. Inf. Sci. 181(6), 1116-1124 (2011)

2. Bonnefon, J.-F., Dubois, D., Fargier, H., Leblois, S.: Qualitative heuristics for balancing the pros and the cons. Theory and Decision 65, 71-95 (2008)

3. Dubois, D., Fargier, H., Bonnefon, J.-F.: On the qualitative comparison of decisions having positive and negative features. J. of Artif. Intellig. Res. 32, 385-417 (2008)

4. Dubois, D., Prade, H.: A theorem on implication functions defined from triangular norms. Stochastica 8, 267-279 (1984)

5. Dubois, D., Prade, H.: Fuzzy rules in knowledge-based systems Modelling gradedness, uncertainty and preference. In: Yager, R.R., Zadeh, L.A. (eds.) An Introduction to Fuzzy Logic Applications in Intelligent Systems, pp. 45-68. Kluwer Acad. (1992)

6. Dvořák, A., Holčapek, M.: Fuzzy integrals over complete residuated lattices. In: Carvalho, J.P., Dubois, D., Kaymak, U., da Costa Sousa, J.M. (eds.) Proc. Joint 2009 Inter. Fuzzy Systems Association World Congress and 2009 Europ. Society of Fuzzy Logic and Technology Conf (ISFA-EUSFLAT), Lisbon, July 20-24, pp. 357-362 (2009) 
7. Grabisch, M.: The Möbius transform on symmetric ordered structures and its application to capacities on finite sets. Discrete Mathematics 287(1-3), 17-34 (2004)

8. Grabisch, M.: The symmetric Sugeno integral. Fuzzy Sets Syst. 139, 473-490 (2003)

9. Grabisch, M., Labreuche, C.: A decade of application of the Choquet and Sugeno integrals in multi-criteria decision aid. Ann. Oper. Res. 175, 247-286 (2010)

10. Greco, S., Matarazzo, B., Slowinski, R.: Bipolar Sugeno and Choquet integrals. In: Proc. EUROFUSE Workshop on Informations Systems, Varenna, Italy, pp. 191-196 (September 2002) 